\title{
One-dimensional periodic metallic structures and its dark hollow lattices by surface plasmons
}

\author{
Yaru Wang ${ }^{1}$, a, Qingya $\mathrm{Liu}^{1}$,Xinzhi Hu${ }^{1}$, Weiyang Tong ${ }^{1}$,Zhengling Wang ${ }^{1}$ \\ ${ }^{1}$ Faculty of Science, Jiangsu University, Zhenjiang 212013, China \\ awyr922119@163.com,
}

\begin{abstract}
Keywords: Metallic structures ; Laser trapping; Surface plasmons
Abstract. We propose a scheme to generate one-dimensional (1D) dark hollow lattices to trap and guide cold atoms periodically at the nanometer scale by surface plasmons (SP) excited on the periodic metallic strips (arrays) with the blue-detuned light. Intensity and optical potential in the trap are calculated by Comsol method, and it shows that closed contour lines of SP intensity and optical potential can realize the effective trapping and guiding of cold atoms in our 1D lattices. Cold atoms are trapped in the region with a section diameter about $30 \mathrm{~nm}$, by which the trap has a large intensity gradient $\left(>10^{13} \mathrm{~W} / \mathrm{m}^{3}\right)$.
\end{abstract}

\section{Introduction}

In recent years, much interest has been directed towards laser trapping and guiding of neutral cold atoms in atomic optics and quantum optics, in which the guiding of cold atoms in a blue-detuned light field, such as dark hollow laser beam [1-2] or blue-detuned LP01-mode evanescent light-wave [3-5] in a hollow optical fiber, a blue-detuned TE01 doughnut mode in a hollow metallic waveguide [6] were studied. In blue-detuned guiding schemes, cold atoms are trapped in the minimum intensity regions of the dark hollow mode and will suffer the minimal light shift and the lowest coherence loss.

With the development of integrated optics, the optical trapping of neutral cold atoms at the nanometer scale below the diffraction limit is becoming a hot research subject in atomic optics and micro-nanooptics. So far, several schemes have been proposed to trap cold atoms at the nanometer scale by the structure of SP. In 2009, Lukin et al. proposed a scheme to trap cold atoms by nanoscale plasmonic structures [7]. In 2009, BrianMurphy et al. proposed a nanoscale electro-optical trap for neutral cold atoms [8]. In 2012, Lukin et al. proposed a scheme of nanoplasmonic lattices for cold atoms by periodic silver nanoshells [9]. In 2013, Wang et al. proposed a scheme of nanoscale guiding for cold atoms based on metallic wedges [10]. In this paper, we propose a scheme to generate 1D dark hollow SP lattices (DHSPL) to trap and guide cold atoms periodically at the nanometer scale by the periodic metallic strips with the blue-detuned light.

\section{Trapping and Guiding scheme and intensity distribution}

A proposed scheme to generate 1D DHSPL to trap and guide cold atoms periodically at the nanometer scale is shown in Fig. 1, in which Fig.1(a) shows schematic diagram of the structure of 1D subwavelength metallic strips (arrays) studied, while Fig.1(b) shows the cross section of the trapping scheme of SP. The structure consists of an infinite dielectric substrate (refractive index n) and metallic arrays formed by periodic rectangular metallic strips in the air atmosphere.

The height and width of the strips are $\mathrm{h}$ and $\mathrm{w}$ respectively and the space between them is $\mathrm{d}$, and the periodicity is the . The coordinate system is set as shown in Fig. 1, where the periodicity is along the $\mathrm{x}$ axis.

When the incident light propagates from the air onto metallic arrays normally, the light field of DHSPL with nanometer scale cross section near the metallic strips will be generated to trap cold atoms with the blue-detuned light. To realize the loading of cold atoms, two-dimensional (2D) moving optical molasses are applied to prepare the MOT and generate a cold atomic beam above DHSPL. Then, atoms can be transferred adiabatically to the blue-detuned light field and guided along it [6]. 

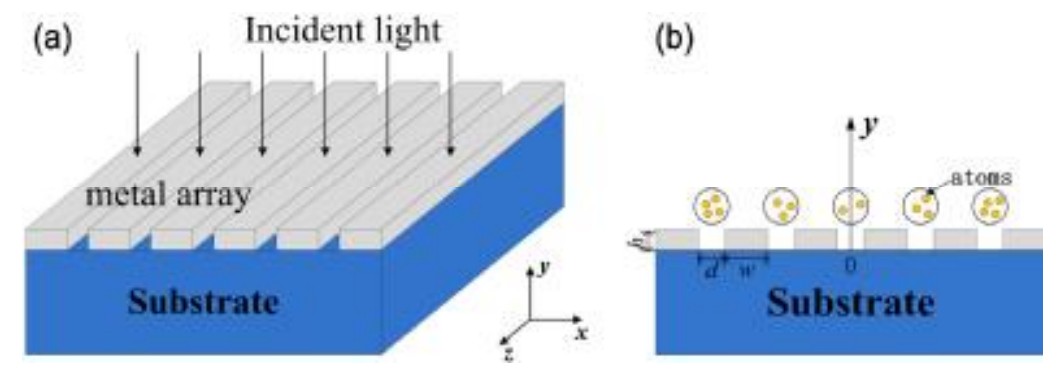

Fig. 1. Scheme of 1D DHSPL. (a) schematic diagram of 1D metallic strips,(b) the cross section of trapping scheme.

By employing the Muti-physics coupling (Comsol) approach, we can obtain the intensity distribution of the DHSPL above 1D subwavelength metallic arrays in Fig.1, the distribution is shown in Fig. 2. For intuition, the distribution of intensity with five periodicities is given in Fig. 2(a), and its distribution of contour lines of intensity is given in Fig. 2(b).

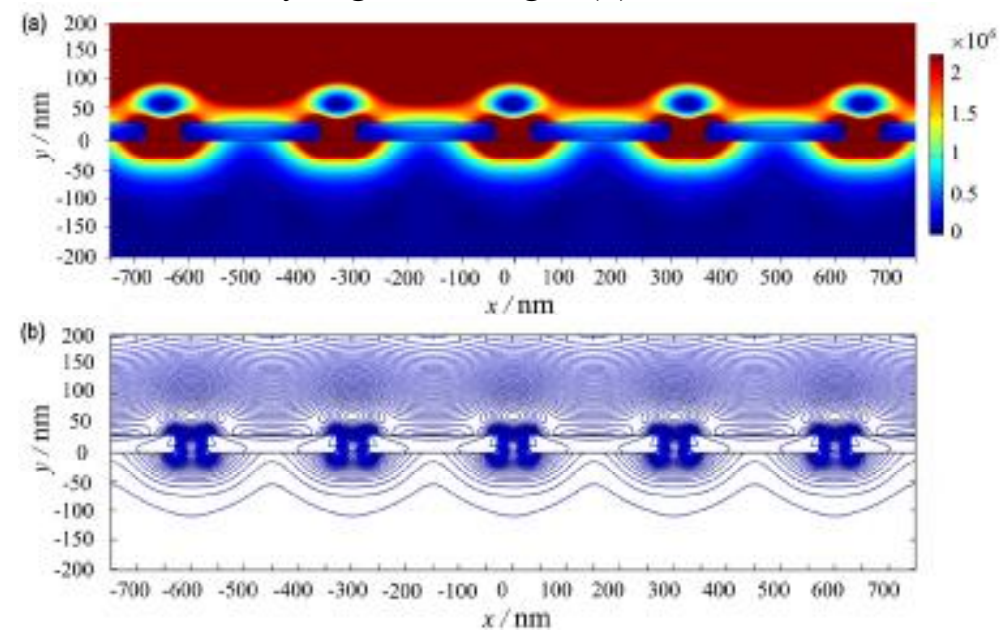

Fig. 2. The intensity distribution of the DHSPL ( unit W/m2 ). (a) the intensity with five periodicities; (b) contour lines of intensity.

As we all know, the Comsol Multiphysics is based on the finite element method, the simulation of real physical phenomena can be realized by solving the partial differential equations (single field) or partial differential equations (multi field) and can obtain the very precise field distribution. Here, we consider a 2D mode in the Comsol to simulate the field distribution near the surface of 1D subwavelength metallic arrays. In simulation, the $\mathrm{z}$ direction in Fig.1. is assumed to be infinite or the length of the metallic arrays is infinite. In fact, if the metallic strips are long enough to make trapping or guiding region much smaller than the length of the metallic strips, 2D mode can be applied to simulate our structure and corresponding error could be neglected.

In our simulation, the metal is silver $(\mathrm{Ag})$, the substrate is quartz glass and its refractive index $n=1.46$, the width and height of rectangular strip are $w=240 \mathrm{~nm}, h=30 \mathrm{~nm}$ respectively. The periodicity of the array $\lambda_{\mathrm{g}}=300 \mathrm{~nm}$, the edge of the rectangular strip is circular with a curvature radius of $5 \mathrm{~nm}$. The wavelength $\lambda$ and the power $P$ of the normal incident light are taken as $0.78 \mu \mathrm{m}$ and $1 \mathrm{mw}$ respectively, and the TM mode of the normal incident light is taken to excite SP and DHSPL. Taking above parameters, 2D distribution of intensity of DHSPL in the $x-y$ plane is simulated, and the result is shown in Fig.2. Here Fig.2 (a) gives the distribution of SP intensity and Fig.2 (b) gives contour lines of intensity. It is obvious that closed periodic dark hollow section SP field at the nanometer scale is formed above the periodical metallic arrays, and it can be used to trap and guide cold atoms with the blue-detuned light at the center of contour lines of intensity. Therefore, DHSPL can be formed by the periodic dark hollow section blue-detuned SP field for cold atoms.

For simplicity and convenience, the enlarged figure for the central trap of DHSPL near the origin point is taken out to be studied in detail, as shown in Fig. 3. Fig. 3(a) shows the 2D distribution of intensity 
of the central trap when $\mathrm{x}$ is between $-75 \mathrm{~nm}$ and $75 \mathrm{~nm}$ in the $x-y$ plane, and Fig. 3 (b) shows its 2D contour lines of intensity. It can be seen from Fig. 3(b) that contour lines are a series of closed circles with the same center at the center of effective trapping region. It is clear that the maximal intensity can reach the order of due to the local enhanced effect of SP, while the minimum intensity can be 0 at the center of the trap. The dark hollow trap has a large intensity gradient $\left(>10^{13} \mathrm{~W} / \mathrm{m}^{3}\right.$, details to be discussed below), which can push cold atoms to the vicinity of the center of the trap with the blue detuned light, and realize the effective trapping of atoms in the in the $x-y$ plane and the effective guiding of atoms along the $\mathrm{z}$ axis.
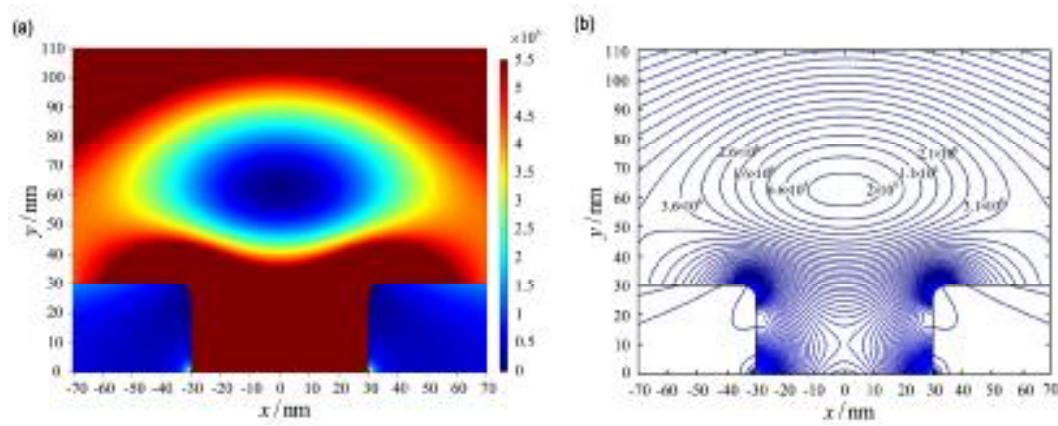

Fig. 3. The enlarged figure for the central trap of DHSPL ( unit W/ m2). (a) the 2D distribution of intensity. (b) contour lines for (a).

\section{Potential analysis and intensity gradient cooling of atoms}

For trapped and guided cold atoms in our DHSPL, we choose the lower hyperfine ground state of the alkali-metal atom as the guided state. Considering the coupling of the three-level atom with a blue-detuned SP field, the expression of the optical potential of atoms in the lower hyperfine ground state can be given by [11]

$$
U_{\text {opt }}=\frac{-\mathrm{h} \delta+\mathrm{h} \sqrt{\delta^{2}+\Omega^{2}}}{2}
$$

Where $\delta / 2 \pi$ is the light detuning from the atomic resonance frequency, $\Omega=\Gamma \sqrt{I / I_{\text {sat }}}$ represents the Rabi frequency, $I_{\text {sat }}$ is the saturation intensity, $\Gamma$ is the natural linewidth, and $I$ is the SP intensity of DHSPL given in Figs. 2 and 3. For ${ }^{87} \mathrm{Rb}-\mathrm{D}_{2}$ line atoms, $\Gamma / 2 \pi=6 \mathrm{MHz}$, and $I_{\text {sat }}=1.67 \mathrm{~mW} / \mathrm{cm}^{2}$. According to Eq. (1), we calculate the distribution of 2D contour lines of optical potential for ${ }^{87} \mathrm{Rb}$ atoms in the $x-y$ plane as $P=5 \mathrm{mw}, \delta / 2 \pi=3 \mathrm{GHz}$, the result is shown in Fig. 4 .

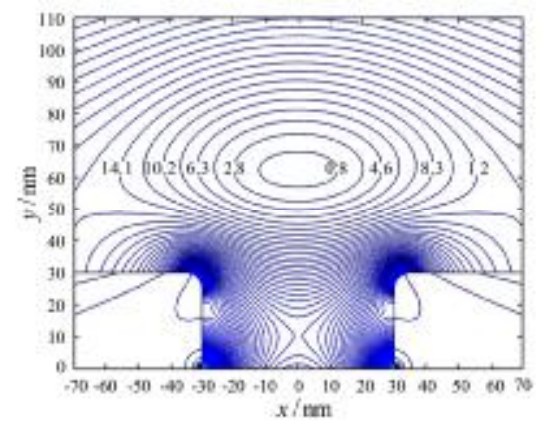

Fig. 4. Closed contour lines of the optical potential at the center trap (unit [mK])

We can find from Fig. 4 that for closed contour lines the optical potential at the center of the trap is zero and the maximum total potential can be $14.1 \mathrm{mK}$, in which cold atoms can be trapped in the vicinity of the center in the $\mathrm{x}-\mathrm{y}$ plane and guided along $z$ axis.

Furthermore, we calculate the 1D distribution of the optical potential for ${ }^{87} \mathrm{Rb}$ atoms in the $\mathrm{x}$ and $\mathrm{y}$ direction through the center of the trap respectively. Fig. 5(a) shows the dependence of the optical potential on $\mathrm{x}$ for different $_{\delta / 2 \pi}$ as $P=5 \mathrm{mw}$, and Fig. 5(b) shows the dependence of the optical potential on x for different $P$ as $\delta / 2 \pi=3 \mathrm{GHz}$. Similarly, Fig. 5(c) shows the dependence of the optical potential on 
y for different $\delta / 2 \pi$ as $P=5 \mathrm{mw}$, and Fig. 5(d) shows the dependence of the optical potential on y for different $P$ as $\delta / 2 \pi=3 \mathrm{GHz}$.
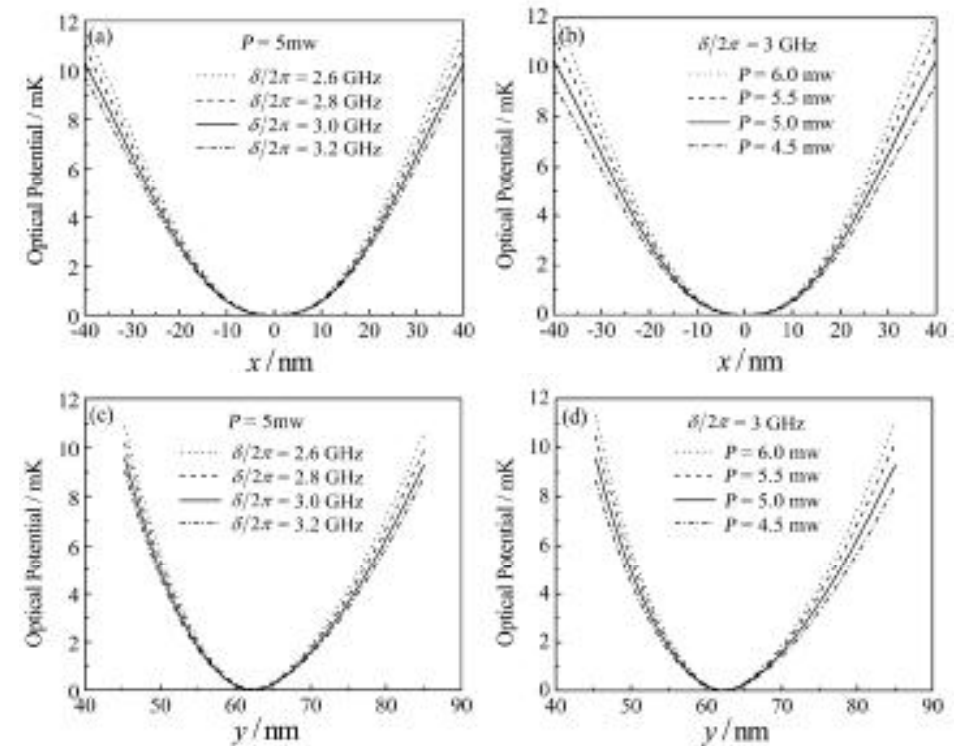

Fig.5. 1D distribution of the optical potential (unit $\mathrm{mK}$ ). (a) optical potential versus $\mathrm{x}$ for different as $\mathrm{P}=5 \mathrm{mw}$; (b) optical potential versus $\mathrm{x}$ for different $\mathrm{P}$ as $=3 \mathrm{GHz}$; (c) optical potential versus $y$ for different as $\mathrm{P}=5 \mathrm{mw}$; (d) optical potential versus y for different $\mathrm{P}$ as $=3 \mathrm{GHz}$.

Here the trapping depth of cold atoms can be defined as the difference between the maximal optical potential and the minimum optical potential in the trap. We find from Fig. 5 that the minimum trapping depth is larger than $9 \mathrm{mK}$ in the $\mathrm{x}$ or $\mathrm{y}$ directions. Therefore, it may trap nearly all atoms $(T=120 \mu \mathrm{K})$ released from the two-dimensional moving optical molasses (MOT) in the x-y plane and guide them along the $\mathrm{z}$ axis. In fact, cold atoms with a temperature of $120 \mu \mathrm{K}$ are trapped in the region with a section diameter about $30 \mathrm{~nm}$ in the trap.

In addition, atoms are not only pushed by the optical potential, but also attracted by the van der Waals attracting potential of metallic strips. The van der Waals attracting potential can be approximately written in the simple form [7]

$$
U_{v d w} \approx-\frac{3}{32} \frac{\mathrm{h} \Gamma}{\left(k_{0} r\right)^{3}}
$$

Where $k_{0}=2 \pi / 0.78 \times 10^{-6}$, and $r$ is the distance from the metallic strips to cold atoms in the trap. For the parameters in Figs. 1 - 5, the minimum distance $r$ from the metallic strips to cold atoms in the trap is about $45 \mathrm{~nm}$, and the van der Waals attracting potential is about $0.56 \mathrm{mK}$. It is obvious that the van der Waals attracting potential is much smaller than the trapping depth $(>9 \mathrm{mK})$, and the van der Waals attracting potential can be negligible in our trap. But the center of trap will be changed a bit $(\sim 7 \mathrm{~nm})$ for the van der Waals attracting potential.

\section{Conclusion}

In conclusion, we have proposed a scheme to generate 1D DHSPL to trap and guide cold atoms periodically at the nanometer scale by the periodic metallic strips with the blue-detuned light. The distribution of SP intensity and optical potential are investigated. It shows that closed contour lines of SP intensity and optical potential can realize the effective trapping of atoms in the in the cross section plane and the effective guiding of atoms along the longitudinal axis. 


\section{Acknowledgements}

Natural Science Foundation of the Jiangsu Province (BK2011462), the Key Project of Natural Science Research of Higher Education Institutions of Jiangsu Province (15KJA140001), the Key Project of college students Innovation and Entrepreneurship Training of Higher Education Institutions of Jiangsu Province (201510299001Z).

\section{References}

[1] J. Yin, W. Gao, and Y. Zhu, "Generation of dark hollow beams and their applications," Prog. Opt. 45, 119-204 (2003).

[2] J. Yin, Y. Zhu, W. Jhe, and Y. Wang, "Atom guiding and cooling in a dark hollow laser beam," Phys. Rev.A 58, 509-513 (1998).

[3] 1. M. J. Renn, E. A. Donley, E. A. Cornell, C. E. Wieman, and D. Z. Anderson, "Evanescent-wave guiding of atoms in hollow optical fibers," Phys. Rev. A 53, R648-R651 (1996).

[4] H. Ito, T. Nakata, K. Sakaki, M. Ohtsu, K. I. Lee, and W. Jhe, "Laser spectroscopy of atoms guiding by evanescent waves in micron-sided hollow optical fibers," Phys. Rev. Lett. 76, 4500-4503 (1996).

[5] H. Ito, K. Sakaki, W. Jhe, and M. Ohtsu, "Evanescent-light induced atom-guidance using a hollow optical fiber with light coupled sideways,” Opt. Commun. 141, 43-47 (1997).

[6] Z. Wang, M. Dai, and J. Yin, "Atomic (or molecular) guiding using a blue-detuned doughnut mode in a hollow metallic waveguide," Opt. Express. 13, 8406-8423 (2005).

[7] D. E. Chang,J. D. Thompson,and M. D. Lukin, “Trapping and Manipulation of Isolated Atoms Using Nanoscale Plasmonic Structures,” Phys. Rev. Lett.103, 123004 (2009).

[8] BrianMurphy, LeneVestergaardHau, "Electro-Optical Nanotraps for Neutral Atoms", Phys. Rev. Lett. 102, 033003(2009).

[9] M. Gullans,T. G. Tiecke,D. E. Chang, and M. D. Lukin, "Nanoplasmonic Lattices for Ultracold Atoms," Phys. Rev. Lett.109,235309(2012).

[10] Z. Wang, W. Tang, M. Zhou, C. Gao, "Nanoscale guiding for cold atoms based on surface plasmons along the tips of metallic wedgesChin", Phys. B 22, 073701 (2013).

[11] J. Yin, "Realization and research of optically-trapped quantum degenerate gases ",Phys. Rep. 430,1-116 (2006). 